\title{
Empreintes génétiques du chou-fleur par RAPD et vérification de la pureté hybride $F_{1}$ d'un lot de semences
}

\author{
S Boury, I Lutz, M-C Gavalda, F Guidet, A Schlesser \\ Groupement d'intérêt publique, Prince de Bretagne biotechnologie, Penn Ar Prat, 29250 Saint-Pol-de-Léon, France
}

(Reçu le 5 mai 1992; accepté le 2 septembre 1992)

\begin{abstract}
Résumé - Les variétés hybrides $F_{1}$ de chou-fleur (Brassica oleracea var botrytis), permettant des récoltes homogènes, sont très appréciées des producteurs. Toutefois, la production de ces semences hybrides est délicate et les lots peuvent être contaminés par des graines issues de l'autofécondation de la lignée parentale femelle. Le taux d'inbreds est donc déterminé avant la commercialisation d'un hybride pour éviter tout accident de récolte. Actuellement, ceci est réalisé par un test phénotypique. Pour gagner du temps et obtenir des résultats non ambigus, nous proposons de le remplacer par une technique récente de biologie moléculaire : la RAPD (random amplified polymorphic DNA) qui correspond à des amplifications par PCR (Polymerase chain reaction) réalisées chacune avec une seule amorce dont la courte séquence a été formulée au hasard. Le but est d'obtenir un marqueur de la lignée parentale mâle qui ne peut être détecté que chez les seuls hybrides. Pour adapter cette technique à notre matériel végétal, nous avons fait varier certains paramètres de PCR. Nous avons ainsi pu constater que la température de l'étape d'hybridation de l'amorce et la concentration de cette dernière influencent les amplifications. La RAPD s'est avéree fiable et rapide comme outil de contrôle de la pureté hybride $F_{1}$ de lots de semences de chou-fleur.
\end{abstract}

Brassica oleracea var botrytis $=$ chou-fleur $/$ hybride $F_{1} / /$ mbred $/$ RAPD $/$ amorce

Summary - Genetic fingerprinting in cauliflower by the RAPD method and determination of the level of inbreeding in a set of $F_{1}$ hybrid seeds. $F_{1}$ hybrid varieties of cauliflower (Brassica oleracea var botrytis), which produce homogeneous crops, are popular with growers. However, the production of these hybrid seeds is difficult and they may be contaminated by inbreds ie seeds arising from self-fertilization of the maternal line. The level of inbred occurrence is therefore determined before marketing the hybrid to avoid problems with the crop. At the moment this is carried out by a phenotypical test. In order to save time and ensure non-ambiguous results, detection of the level of inbreds is proposed via a new molecular biology technique, the RAPD (random amplified polymorphic DNA) method, which is based on PCR (polymerase chain reaction) amplifications each carried out with a single primer, the short sequence of which has been randomly written. The goal is to obtain a marker of the paternal line which can only be detected in hybrids. To adapt this method to our plant material, we tested and modified various PCR parameters. We were thus able to determine that the annealing temperature and the primer concentration act upon amplifications. The RAPD method therefore proved to be a reliable and fast tool for assessing the purity of cauliflower $F_{1}$ hybrid seed.

Brassica oleracea var botrytis $=$ cauliflower $/ F_{1}$ hybrid $/$ inbred $/$ RAPD $/$ primer

\section{INTRODUCTION}

Afin d'améliorer les espèces végétales cultivées, les sélectionneurs utilisent de plus en plus des techniques de biologie moléculaire. En effet, des marqueurs moléculaires peuvent être associés à certains caractères phénotypiques des plantes, permettant alors l'obtention de véritables empreintes génétiques des variétés étudiées. Ainsi, le sélectionneur dispose d'un outil l'aidant au choix des plantes à croiser pour la création de nouvelles variétés. Une sélection rapide et efficace est donc possible grâce au suivi, dans la descendance de ces croisements, de caractères agronomiques parentaux intéressants, avant même leur expression phénotypique.

Le premier outil moléculaire du sélectionneur a été l'étude de familles d'iso-enzymes. Une famille est composée de protéines enzymatiques catalysant la même réaction chimique mais différant, 
par exemple, par leur composition en acides aminés ou leur longueur (Tanksley and Orton, 1983). Mais le polymorphisme enzymatique ne révèle qu'une faible part du polymorphisme génomique, car moins de 10\% de l'ADN végétal code pour des protéines. De nouvelles techniques, étudiant directement la molécule d'ADN, sont ensuite apparues. Elles s'intéressent à la fois aux portions codantes et non codantes du génome. II s'agit de la technique RFLP (Restriction fragment length polymorphism, pour revue : Tanksley et al, 1989) et de la toute récente technique RAPD (Random amplified polymorphic DNA, Williams et al, 1990). La première résulte d'une distribution différente des sites de restriction des endonucléases, d'un génome à un autre, générant un polymorphisme des longueurs des fragments de restriction. La seconde révèle également des variations de séquence nucléotidique entre génomes. Elle correspond à une répartition différente, d'une plante à une autre, des sites d'hybridation d'une courte amorce oligonucléotidique monocaténaire, dont la séquence a été formulée au hasard. Après amplification par PCR (Polymerase Chain Reaction, pour revue : Erlich et al, 1989) avec une amorce de ce type, un polymorphisme des profils électrophorétiques des fragments d'ADN amplifiés est souvent observé entre plusieurs génomes.

Chez beaucoup d'espèces cultivées, la production de variétés hybrides $F_{1}$ s'est développée depuis quelques années car ces dernières fournissent souvent de meilleurs rendements. Ainsi, après les hybrides de chou-fleur d'automne, les premiers hybrides de chou-fleur d'hiver (Brassica oleracea var botrytis) apparaissent sur le marché. Leur homogénéité génétique permet à l'agriculteur de réduire considérablement le nombre de passages nécessaires pour la récolte. La castration manuelle des organes mâles de la lignée femelle n'est pas envisageable chez le chou-fleur dont les fleurs, très nombreuses, sont bisexuées et de petite taille. La production de ces hybrides de chou-fleur repose donc sur l'emploi de lignées mâles-stériles ou autoincompatibles en tant que parents femelles. Ce dernier caractère est le fruit de l'expression d'un locus multiallélique (pour revue : Nasrallah et al, 1991). Cette auto-incompatibilité est rarement stricte et elle peut même être levée lors de conditions climatiques inhabituelles. Des risques de même nature peuvent aussi être encourrus par l'utilisation de certaines stérilités mâles, dont l'expression peut ne pas être parfaite. Dans les 2 cas, ceci est à l'origine d'une contamination plus ou moins importante des lots de semences hybrides par des graines issues de l'autofécondation de la lignée parentale femelle. Or, la variété hybride ne peut être commercialisée qu'avec une très faible contamination. Jusqu'à présent, le taux d'autofécondation résiduel du parent femelle (taux d'inbreds) était évalué par un test phénotypique sur un échantillon d'une centaine de plantes. Afin de distinguer les hybrides et les inbreds, il est souvent nécessaire qu'ils aient atteint un stade de développement suffisant, ce qui constitue une perte de temps pour la commercialisation. La RAPD peut résoudre ce problème en suppléant le test phénotypique par l'analyse moléculaire de très jeunes plants. En effet, le profil électrophorétique des fragments d'ADN amplifiés à partir de l'ADN de la lignée mâle (profil RAPD) peut présenter une ou plusieurs bandes qui le distinguent de celui de la lignée femelle. Le profil RAPD d'une plante dont les parents sont homozygotes doit théoriquement être constitué de l'ensemble des bandes des profils parentaux. Ainsi, le profil d'un hybride $F_{1}$ doit présenter les bandes spécifiques de la lignée parentale mâle contrairement à celui d'un inbred.

Pour optimiser l'obtention de profils RAPD à partir d'ADN de chou-fleur, nous avons fait varier certains paramètres de PCR. Dans cet article, nous décrivons l'influence de ces variations sur les profils RAPD. Nous illustrons l'intérêt de cette technique par une application simple : la détermination du taux d'inbreds d'un lot de semences de chou-fleur supposées hybrides $F_{1}$

\section{MATÉRIEL ET MÉTHODES}

\section{Matériel végétal : choux-fleurs (Brassica oleracea var botrytis)}

L'ensemble du matériel végétal utilisé provient du programme d'amélioration génétique du chou-fleur conduit par I'INRA en collaboration avec les organisations professionnelles bretonnes.

Les lignées $L_{1}$ et $L_{2}$ ont été fournies par l'INRA et nous ont servi pour la mise au point de la méthode RAPD avec de l'ADN de chou-fleur.

Nous avons ensuite déterminé le taux d'inbreds de 2 lots de semences supposées hybrides:

- l'un nous a été fourni par le Groupement malouin de sélection (GMS):

$$
F_{1 G M S}: L_{3} \times L_{4}
$$


- l'autre par l'Organisation bretonne de sélection (OBS)

$F_{10 B S}: L_{2} \times \begin{aligned} & L_{5} \\ & \text { et } \\ & L_{6}\end{aligned}$

Chacun de ces 2 lots était accompagné par une plante de chaque lignée parentale. Les graines ont été semées en «mini-mottes de terreau» placées en serre. Soulignons que 2 lignées sœurs de chou-fleur $L_{5}$ et $L_{6}$ ont servi de parents mâles pour l'hybride fourni par l'OBS. Elles présentent, en effet, le même phénotype et ont des génotypes peu distants, car elles sont issues de la $6^{\mathrm{e}}$ génération d'autofécondation d'une même plante initiale.

\section{Extractions de I'ADN des choux-fleurs}

Les ADN des lignées parentales fournies par l'INRA et le GMS $\left(L_{1}, L_{2}, L_{3}\right.$ et $\left.L_{4}\right)$ ont été extraits selon le protocole de Dellaporta et al (1983) auquel de petites modifications ont été apportées : l'extraction est effectuée à partir de feuilles lyophilisées et I'ADN subit une unique précipitation à l'isopropanol. Cette dernière précède une purification en gradient de $\mathrm{CsCl}$. La bande d'ADN est prélevée et le bromure d'éthidium (BrEt) est éliminé avec du butanol saturé en TE $\left(10 \mathrm{mmol} .^{-1}\right.$ Tris$\mathrm{HCl}, \mathrm{pH}=8,0$, et $1 \mathrm{mmol} . \mathrm{I}^{-1} \mathrm{CTBA}, \mathrm{pH}=8,0$ ) puis I'ADN est précipité par 3 volumes d'éthanol $70 \%$ pendant 30 min à $-20^{\circ} \mathrm{C}$ afin d'éliminer le $\mathrm{CsCl}$. II est finalement remis en suspension dans du TE et sa concentration est alors évaluée au spectrophotomètre (Sambrook et al, 1989). L'ADN des lignées parentales fournies par l'OBS $\left(L_{2}, L_{5}\right.$ et $\left.L_{6}\right)$ a été extrait selon ce même protocole mais avec 2 précipitations successives à l'isopropanol et sans ultracentrifugation en gradient de $\mathrm{CsCl}$ (I'ADN de la lignée $\mathrm{L}_{2}$ extrait ainsi sera noté par la suite $\mathrm{L}_{2}{ }^{*}$ ).

L'ADN des jeunes plantes hybrides $F_{1}$ a été extrait selon la méthode rapide d'Edwards et al (1991). Chaque culot d'ADN a été resuspendu dans $400 \mu \mathrm{l}$ de TE dont seulement $1 \mu \mathrm{l}$ sert à la réalisation d'une amplification RAPD

\section{Oligonucléotides}

Les oligonucléotides des séries SB et JW (tableau I) ont été synthétisés au laboratoire du Dr Ferrec (Centre de transfusion sanguine de Brest). Les séquences des amorces SB ont été conçues au laboratoire et celles de la série JW sont inspirées de Williams et al (1990). Tous ces oligonucléotides, livrés sous forme lyophilisée, ont été remis en suspension dans du TE. Leur concentration a été estimée en considérant qu'une unité de densité optique à $260 \mathrm{~nm}$ équivaut à $33 \mu \mathrm{g}$ d'amorce par $\mathrm{ml}$ (Sambrook et al, 1989). Leur séquence, supérieure à dix nucléotides et d'au moins $40 \%$ de bases $\mathrm{G}+\mathrm{C}$ (limites minimales pour l'obtention de fragments amplifiés en RAPD, Williams et al, 1990), respecte les contingences mirnsées à une amorce de PCR, notamment l'absence de sequiences palindromiques, de polypurines et de polypyrimidines.

Les amorces des séries $C$ et $D$ (tableau I) proviennent de la société Operon (Alameda, USA). Livrées sous forme lyophilisée, elles ont été remises en suspension selon les spécifications du fournisseur.

\section{Amplification de fragments aléatoires d'ADN}

Le protocole d'amplification est une adaptation de celui décrit par Williams et al (1990). Chaque réaction de PCR est réalisée dans un volume de $25 \mu \mathrm{l}$ recouvert de 2 gouttes d'huile minérale. Le milieu d'amplification contient, soit $1 \mu \mathrm{l}$ d'ADN extrait selon Edwards et al (1991) soit $12,5,25$ ou $75 \mathrm{ng}$ d'ADN extrait selon Dellaporta et al (1983). Différentes concentrations en oligonucléotide ont été testées : $0,3,0,8$ et $1,6 \mu \mathrm{mol} .1^{-}$ 1 pour les amorces des séries SB et JW, ou $0,2,0,5$ et $1 \mu \mathrm{mol} . \mathrm{I}^{-1}$ pour celles d'Opéron. Chaque échantillon contient également 1 unité de Taq polymérase (différentes marques ont été essayées), $100 \mu \mathrm{M}$ de chaque nucléotide libre (dATP, dCTP, dGTP, dTTP; Pharmacia), $10 \mathrm{mmol}^{-1}$ de Tris $-\mathrm{HCl}, \mathrm{pH}=8,0,50$ mmol. $1^{-1}$ de $\mathrm{KCl}$ et $1,5 \mathrm{mmol} . \mathrm{I}^{-1}$ de $\mathrm{MgCl}$ 2. Pour les manipulations effectuées avec la Taq polymérase Promega, les $25 \mu \mathrm{l}$ contiennent en plus $0,1 \%$ de Triton $X-100$. Un témoin sans ADN matrice est réalisé lors de chaque manipulation pour révéler d'éventuelles contaminations par de l'ADN exogène.

Les amplifications ont été réalisées dans un appareil DNA thermal cycler 480 (Perkin Elmer Cetus). Les 45 cycles programmés comportent une étape de dénaturation à $94^{\circ} \mathrm{C}$, pendant $1 \mathrm{~min}$ ( $3 \mathrm{~min}$ pour le premier cycle), une phase d'hybridation des amorces à $30{ }^{\circ} \mathrm{C} \mathrm{d}^{\prime} 1 \mathrm{~min}$ également et une étape de polymérisation à $72{ }^{\circ} \mathrm{C}$, durant $2 \mathrm{~min}$. Les transitions entre chaque palier de température sont les plus brèves possibles.

Tableau I. Caractéristiques des oligonucléotides cités dans cette étude

\begin{tabular}{|c|c|c|c|}
\hline $\begin{array}{l}\text { Nom } \\
\text { de } \\
\text { l'amorc }\end{array}$ & $\begin{array}{l}\text { Séquence } \\
\text { nucléotidique } \\
\text { ce }\end{array}$ & $\begin{array}{c}\text { Nb de } \\
\text { qucléotides }\end{array}$ & $\begin{array}{l}\text { Proportion } \\
\text { de bases } \\
\text { GC }\end{array}$ \\
\hline SB1 & GTTAGACCATTC & 12 & $41,67 \%$ \\
\hline SB2 & GTTAGACCAT & 10 & $40 \%$ \\
\hline JW1 & ACCTCGAGCACTGTCT & 16 & $56,25 \%$ \\
\hline JW2 & CGGTCACTGT & 10 & $60 \%$ \\
\hline JW3 & TGCTCACTGA & 10 & $50 \%$ \\
\hline $\mathrm{CO} 2$ & GTGAGGCGTC & 10 & $70 \%$ \\
\hline $\mathrm{CO6}$ & GAACGGACTC & 10 & $60 \%$ \\
\hline D12 & CACCGTATCC & 10 & $60 \%$ \\
\hline D20 & ACCCGGTCAC & 10 & $70 \%$ \\
\hline
\end{tabular}




\section{Electrophorèse des fragments d'ADN amplifiés}

La révélation de la taille des fragments amplifiés se fait par électrophorèse en gel d'agarose $(1,4 \%)$ dans un tampon TAE $1 \times\left(0,04\right.$ mol. $^{-1}$ Tris-acétate et 0,001 mol. I $^{-1}$ EDTA, $\mathrm{pH}=8,0$; Sambrook et al, 1989) et est visualisée sous lumière ultraviolette après coloration au bromure d'éthidium.

\section{RÉSULTATS}

\section{Influence de certains paramètres d'amplification sur les profils RAPD}

Différentes conditions d'amplification ont été testées afin d'optimiser la technique RAPD avec de I'ADN de chou-fleur. Ainsi, nous avons essayé 3 quantités d'ADN matrice $(12,5,25$ ou $75 \mathrm{ng})$ et 3 concentrations en amorce $(0,3,0,8$ et 1,6 $\mu$ mol..$^{-1}$ pour les oligonucléotides des séries SB et JW, et $0,2,0,5$ et $1 \mu \mathrm{mol} . .^{-1}$ pour ceux d'Opéron, fournis en moins grande quantité).

Le profil RAPD d'une plante, obtenu avec un oligonucléotide donné, est resté le même avec les 3 quantités d'ADN matrice utilisées. Ce résultat a été vérifié pour de multiples couples ADNamorce (fig 1).

Nous avons souvent observé que les fragments d'ADN amplifiés sont d'autant plus courts que la concentration en amorce du milieu est forte (fig 2). Ainsi, sur la figure 2a les bandes s'échelonnent de $740 \mathrm{pb}$ (bande peu visible) à $2900 \mathrm{pb}$ pour une concentration de $0,3 \mu \mathrm{mol} . \mathrm{I}^{-1}$ d'oligonucléotide, et de $560 \mathrm{pb}$ à $1600 \mathrm{pb}$ pour 1,6 $\mu \mathrm{mol}^{.1^{-1}}$ d'amorce. La figure $2 \mathrm{~b}$ illustre de la même manière ce phénomène. Compte tenu de ce résultat, nous avons utilisé ces 2 gammes de concentrations d'amorce pour la recherche de marqueurs RAPD de nos différentes lignées de chou-fleur.

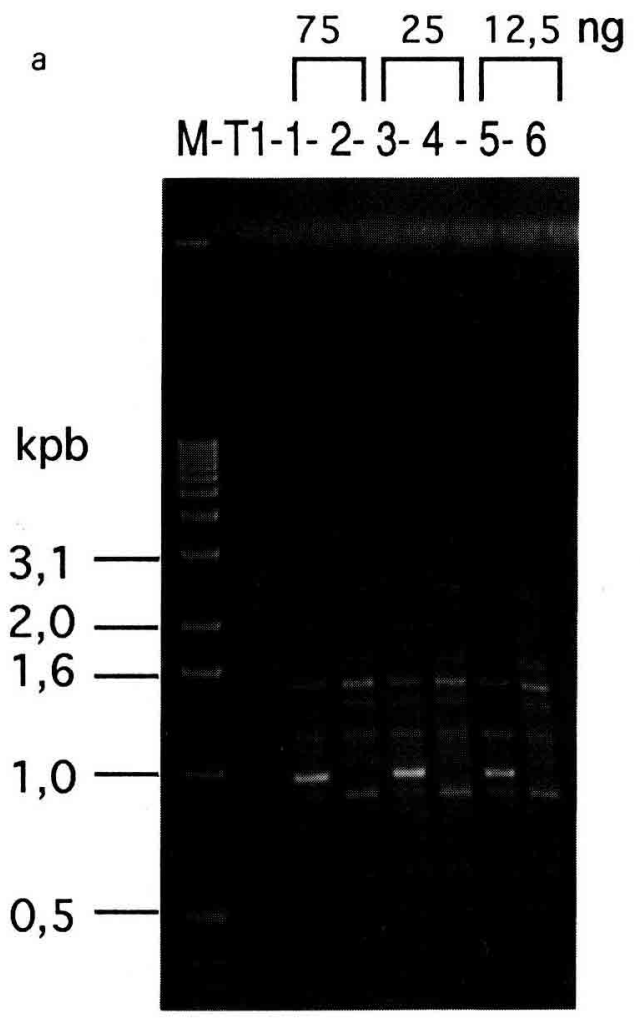

b

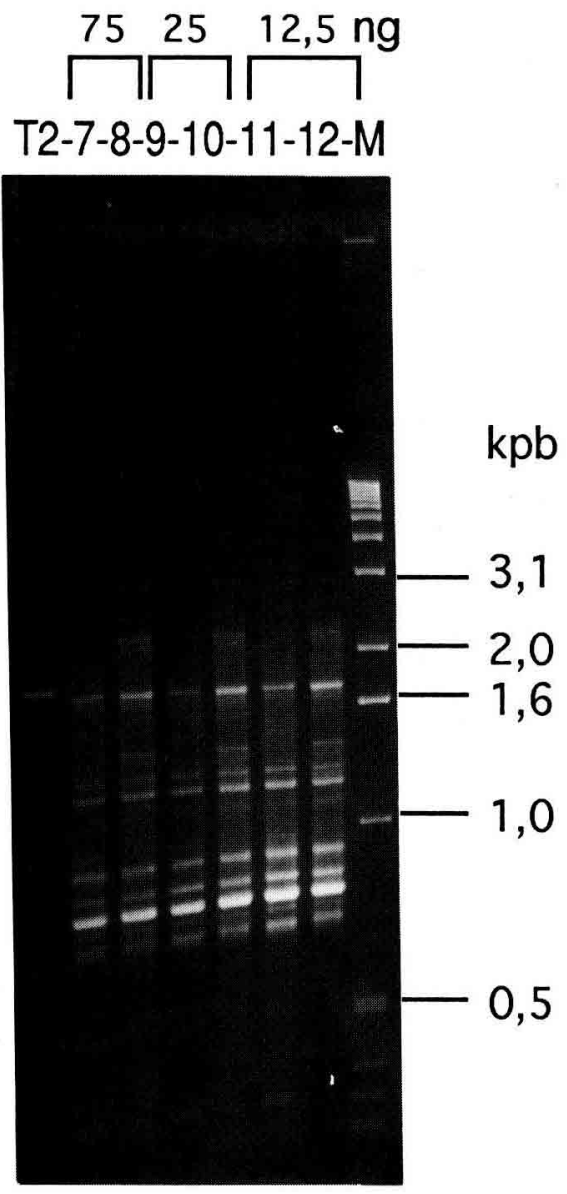

Fig 1. Amplifications avec différentes concentrations d'ADN matrice. Amplifications réalisées avec 1,6 $\mu$ mol. I $^{-1}$ d'amorce (a, SB2 et b, JW2). ADN matrice : ADN des lignées $L_{1}$ (pistes 2, 4, 6, 8, 10 et 12) et $L_{2}$ (pistes 1, 3, 5, 7, 9 et 11) en quantités variables : $75 \mathrm{ng}$ (pistes 1, 2, 7 et 8), $25 \mathrm{ng}$ (pistes 3, 4, 9 et 10) ou 12,5 $\mathrm{ng}$ (pistes 5, 6, 11 et 12). Témoins sans ADN matrice : avec SB2, piste $\mathrm{T}_{1}$, et avec JW2, piste $T_{2}$. Marqueur de masses moléculaires (pistes $M ; 1$ kb DNA ladder; Gibco BRL). 


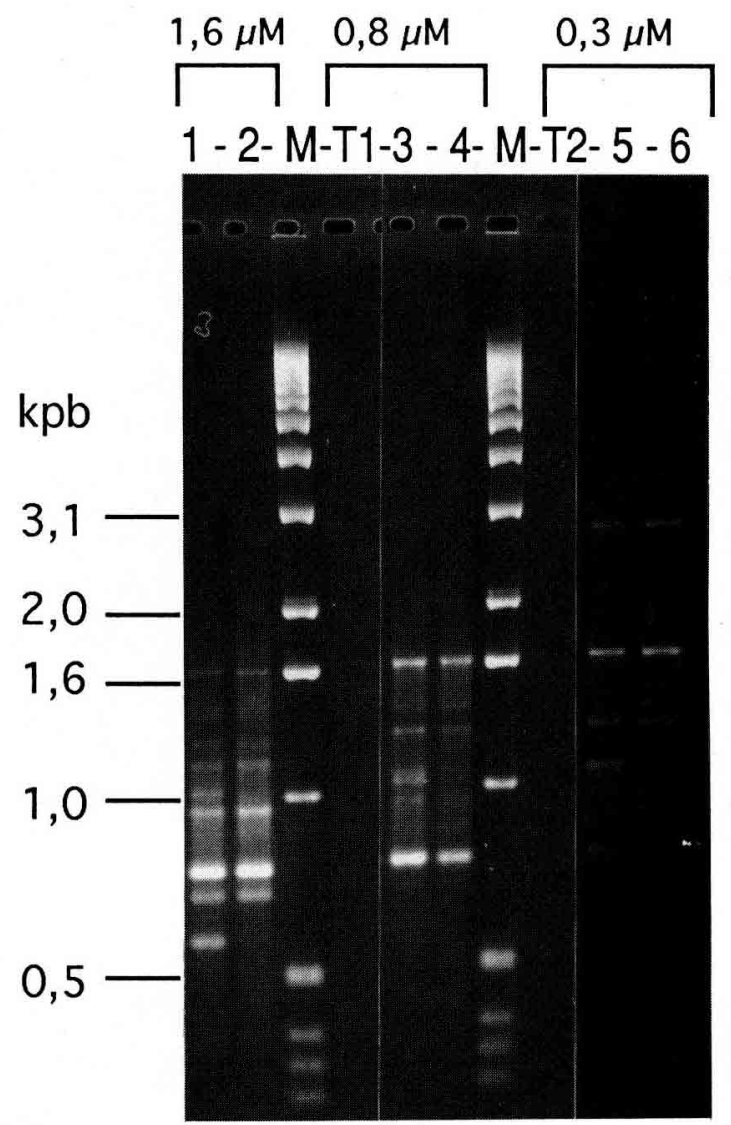

b

concentration en JW 1

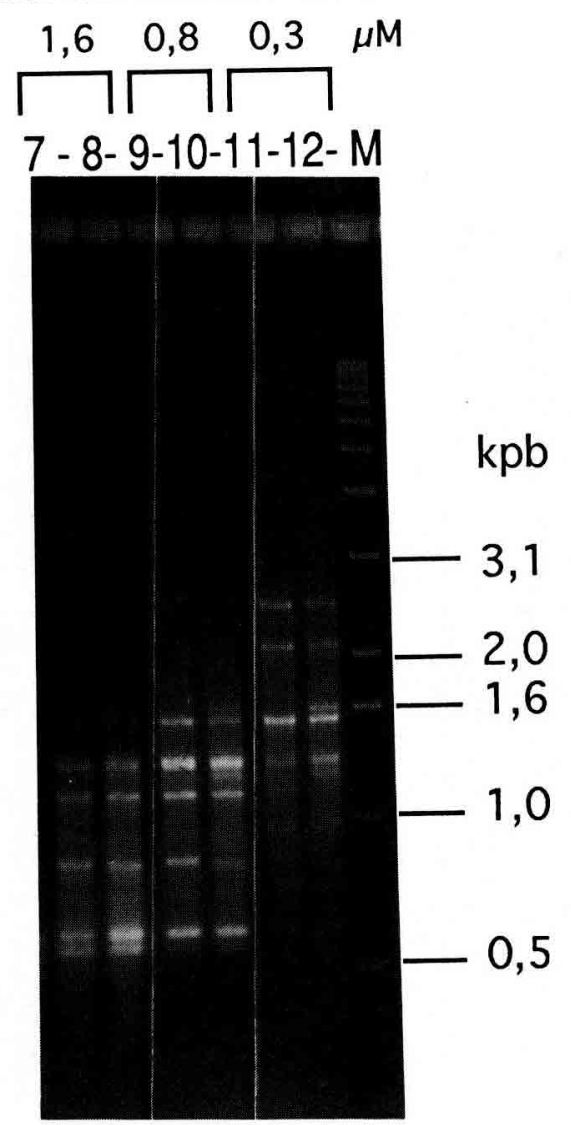

Fig 2. Influence de la concentration en amorce sur un profil RAPD. Amplifications réalisées avec une concentration en amorce de

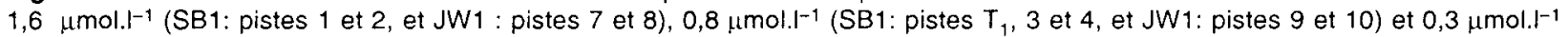
(SB1: pistes $T_{1}, 5$ et 6 , et JW1: pistes 11 et 12). PCR à partir de $25 \mathrm{ng}$ d'ADN matrice des lignées $L_{1}$ (pistes 2,4 et 6 ) et $L_{2}$ (pistes 1,3 et 5), et des lignées $L_{3}$ (pistes 8,10 et 12) et $L_{4}$ (pistes 7,9 et 11). Témoins sans ADN matrice (pistes $T_{1}$ et $T_{2}$ ). Marqueur de masses moléculaires (pistes $M ; 1$ kb DNA ladder; Gibco BRL).

Une modification de la température de l'étape d'hybridation de l'amorce peut changer le profil RAPD obtenu avec un couple ADN matriceamorce. En effet, en passant de $36{ }^{\circ} \mathrm{C}$, température utilisée par Williams et al (1990), à $30^{\circ} \mathrm{C}$, nous avons observé une variation de l'intensité de certaines bandes et l'apparition de nouveaux fragments d'ADN amplifiés (fig 3). Nous avons alors opté pour cette seconde température.

Nous avons également testé différentes Taq polymérases disponibles sur le marché. Nous avons retenu celle de la société Promega qui présente, selon nous, un excellent rapport qualité/prix. D'autres polymérases donnent de très bons résultats mais leur prix reste élevé. Avec certaines amorces, l'une des enzymes testées n'a pas permis l'amplification des fragments d'ADN obtenus avec les autres polymérases, à partir d'un même ADN matrice (résultats non présentés).

\section{Application agronomique de la technique RAPD chez le chou-fleur : détermination du taux dinbreds dans un lot de semences supposées hybrides $F_{1}$}

Lors de la détermination d'un taux d'inbreds, nous avons recherché, dans un premier temps, une amorce générant un marqueur RAPD de la lignée parentale mâle, puis nous avons analysé le génome des jeunes plants supposés hybrides.

Pour l'hybride $F_{1 G M S}$, nous avons testé 14 amorces sur les ADN des lignées parentales $L_{3}$ et $L_{4}$. Quatre d'entre elles (JW2, JW3, C02 et C06) ont permis de caractériser la lignée parentale mâle $L_{4}$, chacune avec un marqueur RAPD. Seules $\mathrm{C} 02$ et C06 en ont généré un de forte intensité, donc facilement reproductible. Ces 2 marqueurs nous ont permis de déterminer un taux d'inbreds de $54 \%$ sur un lot de 100 plants 


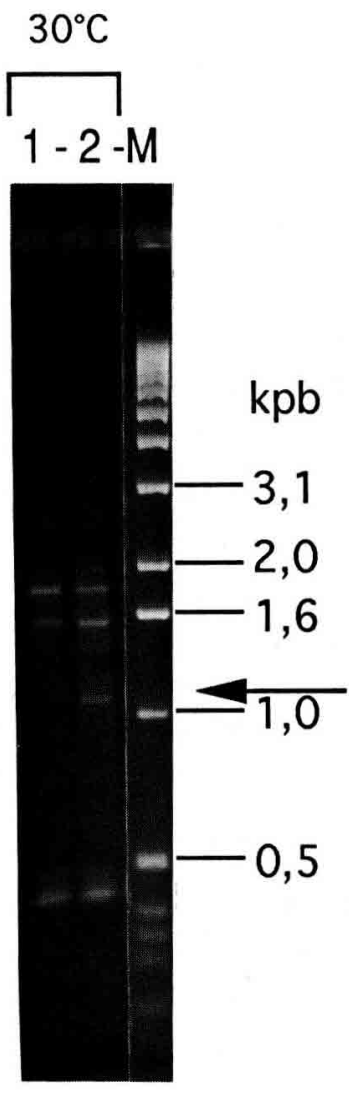

1- 2-3- 4- 5- 6-M-7-8-9-10-11-12-13- 어-M

a

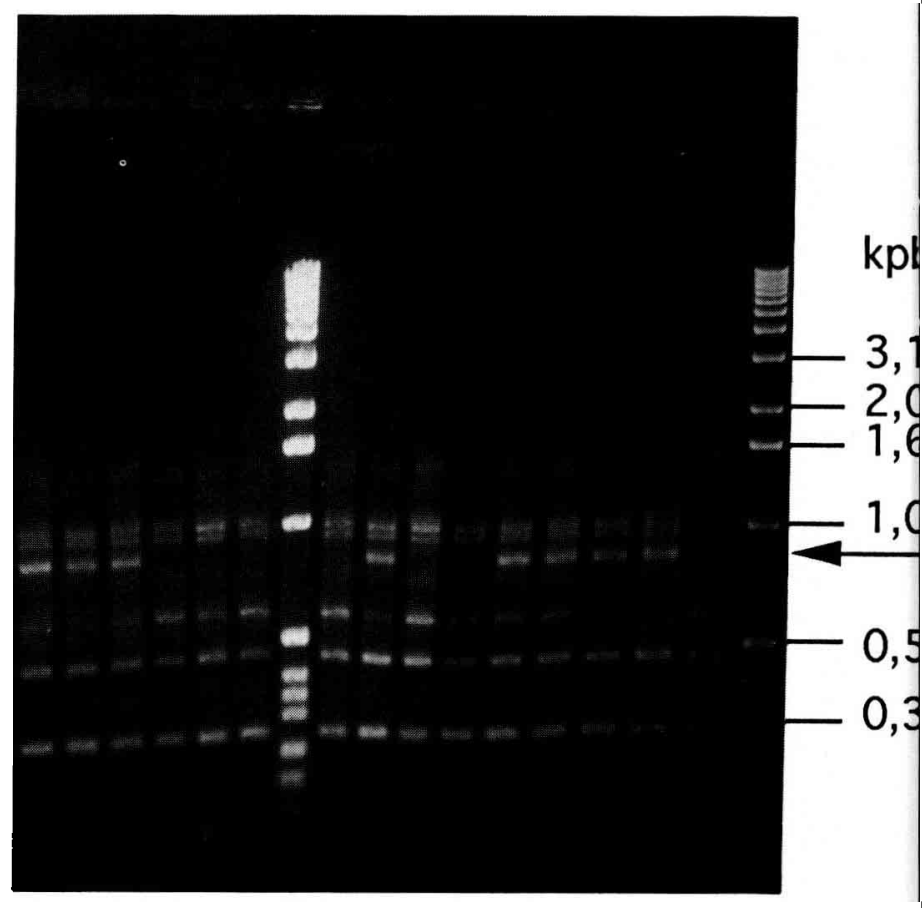

1- 2- 3- 4- 5- 6-M-7-8-9-10-11-12-13- 요요-M

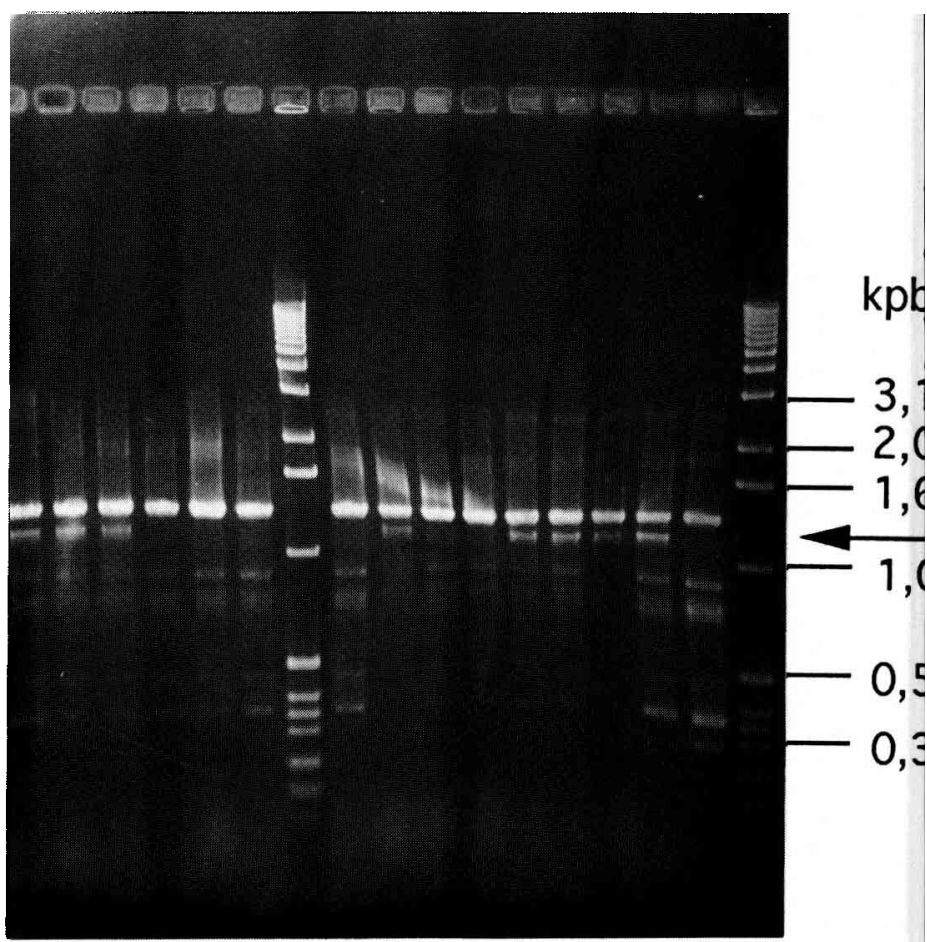

Fig 4. Distinction entre individus hybrides et inbreds d'un lot de semences supposées hybrides $F_{1}$ à l'aide de 2 marqueurs RAPD de la lignée parentale mâle. Amplifications réalisées avec des concentrations en amorce de $1 \mu$ mol..$^{-1}$ pour $\mathrm{CO}_{2}$ (fig 4a) et de $0,5 \mu \mathrm{mol}^{-1^{-1}}$ pour C06 (fig $4 \mathrm{~b}$ ), et à partir de $25 \mathrm{ng}$ d'ADN matrice pour les lignées $L_{3}$ (pistes ) et $L_{4}$ (pistes ) ou à partir de $1 \mu l$ d'ADN extrait par la méthode d'Edwards et al (1991) pour les individus supposés hybrides (pistes 1 à 13). Marqueur de masses moléculaires (pistes $M$; 1 kb DNA ladder; Gibco BRL). Les positions des marqueurs RAPD de la lignée parentale mâle sont indiquées par les flèches.Les individus $1,2,3,8,11,12$ et 13 sont des hybrides contrairement aux numéros $4,5,6,7,9$ et 10 (figs $4 a$, b). 
En vue de généraliser la pratique de la technique RAPD pour déterminer le taux d'inbreds d'un lot de semences hybrides, un second croisement a été étudié ( $\left.F_{10 B S}\right)$. De nouveau, 14 amorces ont été employées afin de trouver des marqueurs des 2 lignées mâles $L_{5}$ et $L_{6}$. L'amorce $D_{12}$ a fourni un marqueur spécifique de ces 2 lignées, tandis que $D_{20}$ a généré un marqueur caractérisant seulement la lignée mâle $L_{5}$. Nous avons vérifié la fiabilité de nos 2 marqueurs à l'aide de 10 plantes phénotypiquement déterminées : 5 hybrides et 5 inbreds (fig 5). Avec l'amorce $D_{12}$, tous les hybrides et tous les inbreds ont été correctement caractérisés (fig $5 \mathrm{a}$ ). Nous avons alors déterminé un taux d'inbreds de $14 \%$ pour ce croisement, dans un lot de 100 jeunes plants supposés hybrides. Avec $D_{20}$, le marqueur du parent mâle $L_{5}$ n'a pas été détecté avec I'ADN des inbreds mais est présent pour 2 hybrides sur 5 (fig 5b).

a
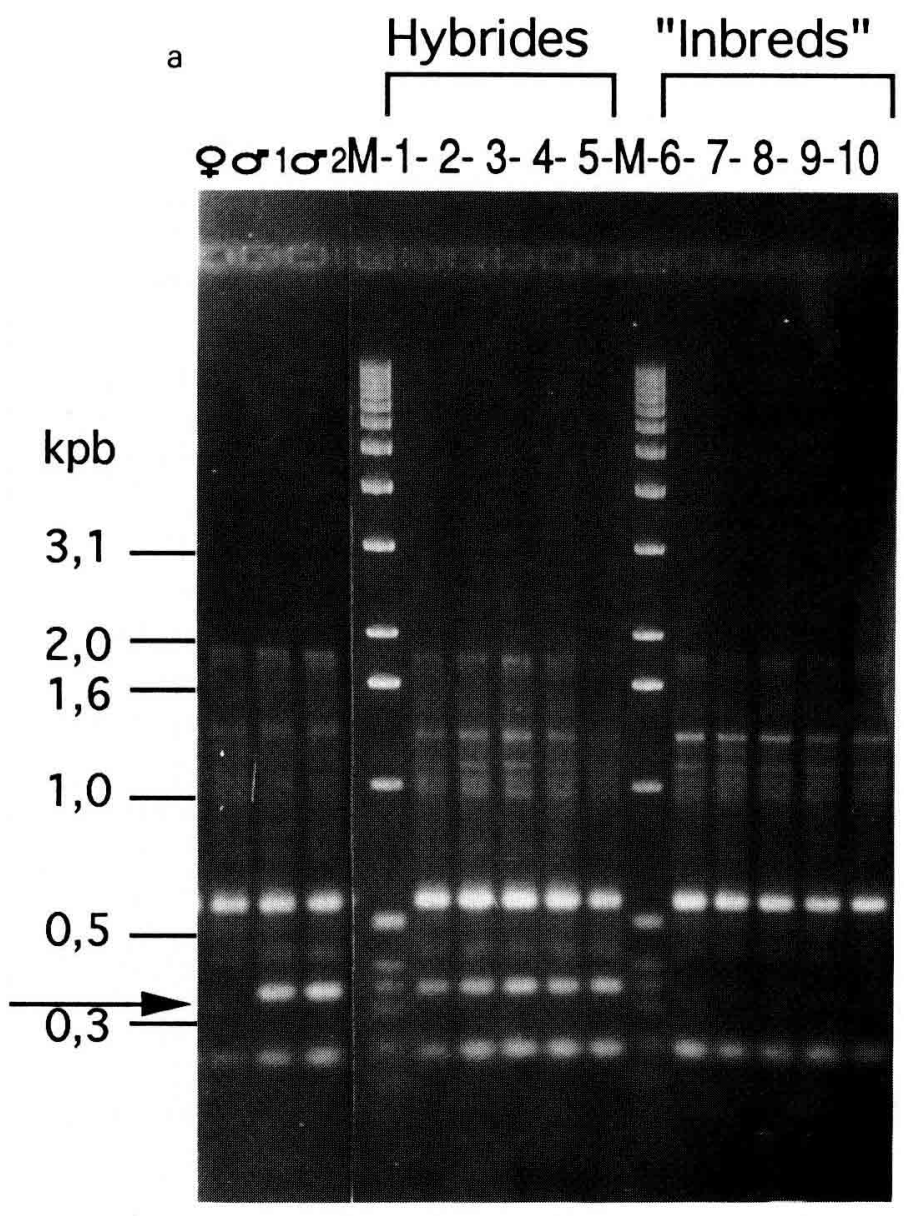

\section{DISCUSSION}

\section{Notions de profil RAPD et de marqueur RAPD}

Nous définissons un profil RAPD comme l'ensemble des fragments d'ADN amplifiés à partir d'un ADN matrice, par PCR avec une amorce (éventuellement plusieurs) de séquence aléatoire, et visualisés après une électrophorèse en gel d'agarose. Certains de ces fragments peuvent distinguer les ADN de différentes plantes et ainsi révéler le polymorphisme de l'ADN au sein de l'espèce étudiée. Nous qualifions ces bandes de marqueurs RAPD.

Au cours de ces travaux, nous avons observé que les bandes peu intenses d'un profil RAPD, correspondant donc aux fragments les moins

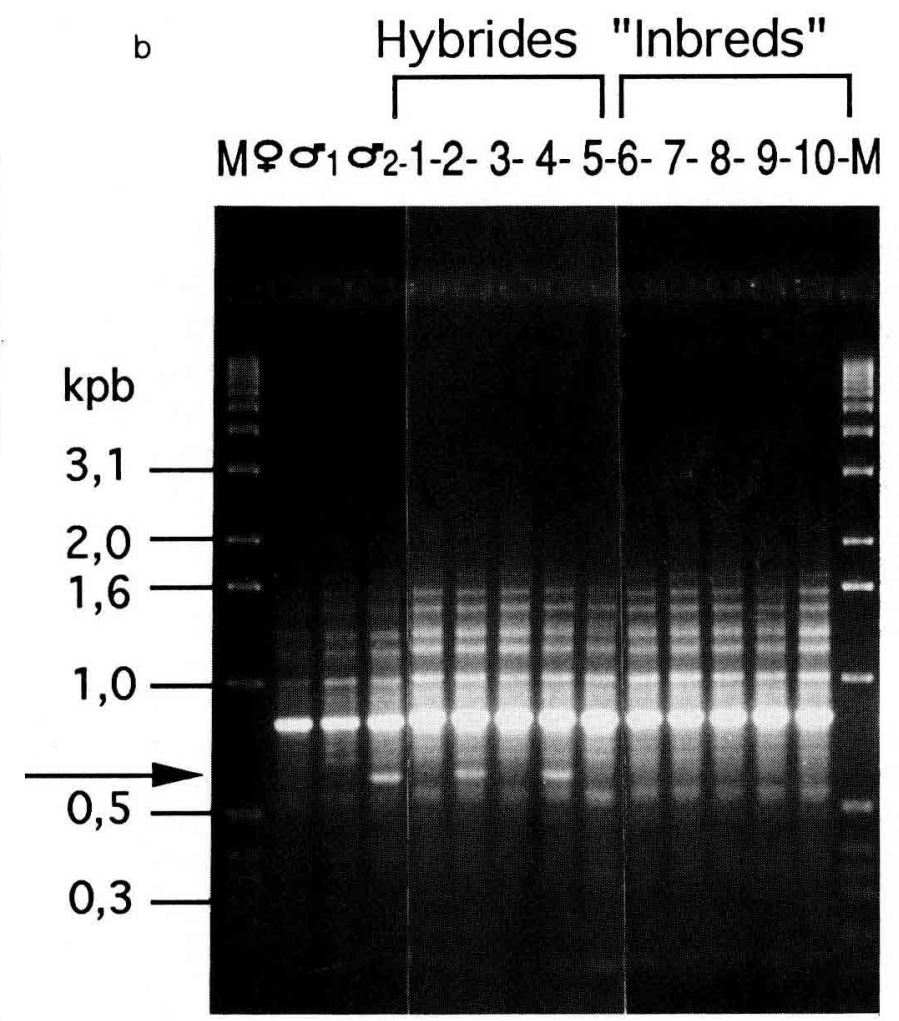

Fig 5. a: Vérification de la valeur d'un marqueur RAPD sur des plantes adultes reconnues phénotypiquement hybrides et inbreds en plein champ. b: distinction entre les 2 lignées paternelles avec un marqueur RAPD permettant de distinguer 2 catégories d'hybrides. Amplifications réalisées avec une concentration en amorce de $1 \mu \mathrm{mol} . \mathrm{I}^{-1}(\mathrm{D} 12$, fig $5 \mathrm{a}$, et D20, fig $5 \mathrm{~b}$ ) et à partir de $25 \mathrm{ng}$ d'ADN matrice pour les lignées $L_{2}{ }^{*}$ (piste ),$L_{5}$ (piste 6 ) et $L_{6}$ (piste 1 ), ou à partir de $1 \mu l$ d'ADN extrait par la méthode d'Edwards et al (1991) pour les individus reconnus phénotypiquement hybrides et inbreds (pistes 1-10). Les positions des marqueurs RAPD des lignées parentales mâles sont indiquées par des flèches. Marqueur de masses moléculaires (pistes M; $1 \mathrm{~kb}$ DNA ladder; Gibco BRL). Les individus 1-5 sont hybrides et ceux de 6-10 sont inbreds aussi bien par le test en plein champ que par l'analyse RAPD (fig 5a). Les individus 2 et 4 sont les "fils" du $d 2$ contrairement aux numéros 1,3 et 5 qui sont «fils" du $\delta 1$ (fig $5 b$ ). 
amplifiés, sont parfois difficilement reproductibles. Nous préférons donc utiliser des marqueurs RAPD de forte intensité. D'autre part, nous pensons qu'il est préférable de ne s'intéresser qu'à des marqueurs situés au milieu des profils RAPD, car nous avons parfois observé des fluctuations concernant les bandes des extrémités de ces profils. Ainsi, en l'absence du marqueur dans un profil, la présence des fragments amplifiés l'encadrant ne laisse subsister aucun doute quant au génotype de la plante étudiée (fig 4 et 5a).

\section{Hérédité des profils RAPD}

Nous avons constaté que, d'une façon générale, toutes les bandes des profils RAPD des 2 lignées parentales homozygotes se retrouvent dans celui de l'hybride $F_{1}$ (fig 4 et 5 ). Cette transmission héréditaire des bandes est une condition indispensable à l'utilisation des marqueurs RAPD en sélection.

Nous avons parfois observé que des jeunes plants présentaient un profil RAPD se distinguant de celui des autres plants supposés hybrides et comportant une ou plusieurs bandes absentes des profils parentaux. Ces individus étaient très probablement issus de graines, soit étrangères au lot étudié, soit résultant d'une fécondation par du pollen contaminant. Ces 2 événements ont une probabilité non nulle, car les graines de chou-fleur sont très petites, donc s'éparpillent facilement, et la pollinisation est réalisée par les abeilles dont les mouvements sont difficilement maîtrisables.

\section{Influence de la concentration en oligonucléotide sur un profil RAPD}

Nos travaux ont montré que plus la concentration en oligonucléotide est forte, plus de courts fragments d'ADN sont amplifiés au détriment des grands qui disparaissent (fig 2). Ce résultat peut s'interpréter en supposant que les hybridations de l'amorce sur I'ADN matrice sont plus nombreuses lorsqu'elle est en forte concentration, générant ainsi des fragments amplifiés plus petits. Ces sites d'hybridation supplémentaires sont très vraisemblablement moins homologues de l'oligonucléotide que ceux obtenus lorsque sa concentration est faible. Si l'explication la plus simple de la disparition d'un grand fragment est
I'hybridation d'une amorce sur un nouveau site se situant au cœur de ce fragment, il est alors surprenant que tous les grands fragments disparaissent. II serait donc intéressant d'isoler et de marquer radio-activement ou chimiquement quelques-un de ces grands fragments. Ainsi, en les utilisant comme sonde, nous pourrions savoir si leur disparition, lorsque la concentration en amorce est forte, résulte de leur non amplification ou de leur amplification en de plus petits fragments.

En faisant varier la concentration en amorce, de grandes différences de tailles entre fragments amplifiés ont pu être obtenues pour un même couple ADN matrice-oligonucléotide : notre plus petit fragment a été de $210 \mathrm{pb}$ (fig $5 \mathrm{a}$ ) et notre plus grand a été de $3400 \mathrm{pb}$ (fig 1a). Toutefois, des tailles très différentes de fragments amplifiés ont été également observées dans un même profil RAPD (fig 1a: de $525 \mathrm{pb}$, bande peu visible, à $3400 \mathrm{pb}$, avec I'ADN de la lignée L1). La recherche d'un polymorphisme entre des ADN différents peut donc être réalisée à la fois sur des grands et sur des petits fragments amplifiés, à l'aide de différentes concentrations en oligonucléotide.

Cette influence de la concentration en amorce pourrait expliquer que les techniques d'AP-PCR (Arbitrarily primed PCR) et de DAF (DNA amplification fingerprinting) utilisent de fortes concentrations d'amorce $\left(10 \mu \mathrm{mol} . \mathrm{I}^{-1}\right.$ pour l'AP-PCR, Welsh and McClelland, 1990, 1991; Welsh et al, 1991a, b; ou environ $3 \mu \mathrm{mol} . \mathrm{I}^{-1}$ pour la DAF Caetano-Anollés et al, 1991). En effet, ces 2 méthodes révèlent souvent des amplifications de très courts fragments. Toutefois, ceci est également lié à la sensibilité des méthodes de révélation (gel d'acrylamide et coloration au nitrate d'argent pour la DAF et autoradiographie après incorporation d'un nucléotide radio-actif pour I'AP-PCR) bien meilleure qu'en RAPD (gel d'agarose coloré au BrEt).

\section{Importance de la température de l'étape d'hybridation de l'amorce}

En abaissant la température de l'étape d'hybridation de l'amorce au cours des cycles de PCR, nous avons constaté que de nouvelles bandes pouvaient apparaître au sein d'un profil RAPD (fig 3). Ce phénomène s'expliquerait par l'hybridation de l'oligonucléotide en de nouveaux sites moins homologues, car la non spécificité est fa- 
vorisée dans ces nouvelles conditions. Selon nous, le même type d'observation doit pouvoir être réalisé entre des profils RAPD obtenus avec des appareils de PCR différents, toute chose égale par ailleurs. En effet, le résultat d'une amplification ne dépend pas que des paliers de température constituant chaque cycle, mais aussi de la durée des transitions entre ces paliers. Or, d'un appareil à un autre, selon les performances des groupes chauffant et réfrigérant, ce sont les durées de ces transitions qui varient le plus, et non la température des paliers. Ainsi, certains ont augmenté le temps pour atteindre $72{ }^{\circ} \mathrm{C}$, en vue d'optimiser leurs amplifications (KleinLankhorst et al, 1991).

\section{Quantité d'ADN matrice}

Comme nous avons fait varier la concentration en ADN matrice d'un facteur 6 (12,5-75 ng) sans observer de modifications des profils RAPD, il semble que ce facteur n'influence pas sérieusement ces derniers. Ainsi, le protocole d'amplification de Quiros et al (1991) emploie des quantités d'ADN qui varient de 25 à $60 \mathrm{ng}$. Toutefois, en augmentant cette échelle de variation, des fluctuations des profils seraient vraisemblablement observables avec des ADN de choux-fleur, comme l'ont montré Carlson et al (1991) avec des ADN de conifères, ainsi que Welsh et McClelland (1990) avec de I'ADN de Staphylococcus aureus. Cette faible sensibilité des profils RAPD à de petites variations de la quantité d'ADN matrice est un avantage. En effet, cela nous a permis de travailler avec des ADN extraits selon la méthode d'Edwards et al (1991) sans même connaître leur concentration. Ceci représente un sérieux gain de temps lors de l'analyse en série de plantes.

\section{Importance de la purification de I'ADN matrice}

Nos travaux ont permis de constater qu'une purification de l'ADN, même succincte, suffit pour l'amplification de fragments d'ADN. Afin de mettre au point la technique RAPD chez le choufleur, nous avions d'abord utilisé des ADN purifiés par ultracentrifugation en gradient de $\mathrm{CsCl}$. Puis nous avons vérifié que le profil RAPD était conservé avec ces mêmes $A D N$, mais extraits sans gradient de $\mathrm{CsCl}$ ni phénol (Edwards et al,
1991). Nous envisageons à l'avenir d'appliquer la technique de Berthomieu et Meyer (1991) qui réalisent une amplification par PCR directement à partir d'un fragment de feuille. Ce dernier point s'inscrit dans une approche visant à réduire la durée de la manipulation pour en augmenter l'efficacité et en diminuer le coût.

\section{Problème des contaminations par de I'ADN étranger et précautions expérimentales}

Lors de chaque manipulation, un échantillon rassemblant tous les constituants de la RAPD, en dehors de I'ADN matrice, a servi de témoin visà-vis d'éventuelles contaminations. Les résultats obtenus avec ces témoins sont de 2 types : soit aucune bande n'est apparue sur le gel (fig 1a et 2a), soit quelques bandes ont été visibles (fig 1b; Williams et al, 1990; Klein-Lankhorst et al, 1991). Même dans ce second cas, il a été conclu que les autres échantillons contenant de I'ADN de chou-fleur n'avaient pas subi de contamination, car leur profil RAPD n'a jamais présenté les bandes du témoin. S'agit-il de contaminations internes des oligonucléotides qui ne pourraient plus se manifester en présence de I'ADN de chou-fleur, celui-ci étant en excès dans l'échantillon de PCR?

Tout problème lié à des contaminations a donc été évité malgré nos faibles précautions: manipulation sans gants et préparation des échantillons hors de toute hotte. Toutefois, nous avons utilisé du matériel de laboratoire autoclavé et 2 jeux de micropipettes : un pour les extractions d'ADN et la préparation des échantillons RAPD, et l'autre pour leur dépôt sur gel après l'amplification par PCR.

\section{Efficacité de la RAPD chez le chou-fleur}

Nos travaux et ceux réalisés dans le même temps par Hu et Quiros (1991) ont montré que le polymorphisme de l'ADN du chou-fleur est facilement accessible par la technique RAPD. En effet, sur les 2 groupes de 14 amorces testées, 7 ont permis de distinguer les lignées parentales de l'hybride $F_{1 G M S}$, et 5 dans le cas de celles de I'hybride $F_{1 O B S}$. Ceci correspond, respectivement, à des taux de $50 \%$ et de $36 \%$ de réussite pour la découverte de marqueurs RAPD caractérisant les lignées parentales de ces 2 croisements. Avec les lignées $L_{1}$ et $L_{2}$, sur les 8 
amorces testées, 6 ont fourni au moins un marqueur RAPD de l'une des lignées, soit un taux de réussite de $75 \%$. Ce résultat est nettement supérieur aux 2 précédents, car ces 2 lignées sont très distantes génétiquement.

L'efficacité de la RAPD pour détecter un polymorphisme de l'ADN est démontrée par l'hybride $F_{1 O B S}$, dont les 2 lignées parentales mâles, bien que sœurs et issues de 6 autofécondations successives, ont été distinguées par un marqueur.

Étant donné les contingences imposées à une amorce RAPD (une dizaine de nucléotides, environ $50 \%$ de bases G-C et pas de séquence palindromique), le nombre d'oligonucléotides utilisables est quasiment infini. De plus, l'utilisation des amorces en couple (Welsh and McClelland, 1991; Klein-Lankhorst et al, 1991) et en gamme de concentrations multiplie les possibilités. La RAPD semble donc très prometteuse en amélioration du chou-fleur.

\section{RAPD et analyse iso-enzymatique pour la détermination du taux d'inbreds d'un lot de semences hybrides}

Pour l'espèce Brassica oleracea, 9 systèmes iso-enzymatiques sont utilisés (Arus et al, 1982; Arus and Orton, 1983; Boucault, 1990) et ont été proposés afin d'évaluer la pureté hybride d'un lot de semences (Tanksley and Orton, 1983). Chacun de ces systèmes présente 1-3 loci polymorphes avec 2-4 allèles par locus (Boucault, 1990). Ce nombre d'allèles disponibles n'est vraisemblablement pas suffisant pour caractériser chaque lignée de chou-fleur, existante ou en cours d'obtention. Ainsi, dans le cas de certains hybrides, on peut envisager que les isozymes soient inefficaces pour caractériser leur lignée parentale mâle, ne permettant alors pas la détermination du taux d'inbreds. Ce cas ne doit pas pouvoir se présenter avec la technique RAPD pour laquelle le nombre d'amorces utilisables est quasiment infini et permet, en théorie, de couvrir tout le génome de l'espèce étudiée

Grâce à la rapidité du protocole d'extraction d'ADN décrit par Edwards et al (1991), la technique RAPD rivalise avec l'analyse isoenzymatique dont le protocole d'extraction est très simple. De plus, le contenu en protéines d'une cellule dépend généralement du tissu et de l'organe auxquels elle appartient. Ainsi, d'une plante à une autre, les protéines doivent être extraites d'un même organe au même stade physiologique et dont le contenu protéique est peu sensible aux variations du milieu. Ce problème ne se pose pas avec la RAPD car toutes les cellules d'une plante, quel que soit son stade de développement, possèdent théoriquement le même génome.

Pour révéler une activité enzymatique, il est nécessaire que la protéine dont elle résulte soit active. L'électrophorèse iso-enzymatique doit donc respecter cette activité par une migration dite "en conditions natives" (respect des différents niveaux de structure des enzymes). Ainsi, tous les systèmes iso-enzymatiques ne requièrent pas le même $\mathrm{pH}$ d'électrophorèse. Les gels d'électrophorèse employés pour distinguer les différents allèles des loci iso-enzymatiques étudiés sont soit en amidon soit en acrylamide selon le pouvoir résolutif nécessaire. Chaque système iso-enzymatique possède son propre système de révélation. Malgré ces 3 dernières contingences, il est toutefois possible de révéler différentes activités enzymatiques à partir d'un même gel de protéines, si ces enzymes supportent le même $\mathrm{pH}$ d'électrophorèse et si leurs différentes formes se distinguent dans un gel d'amidon. II faut alors couper délicatement ce gel dans son épaisseur en plusieurs tranches dont le nombre correspond à celui des systèmes iso-enzymatiques testés et chaque tranche doit subir son propre protocole de révélation. La RAPD semble donc plus facile d'emploi pour la recherche en routine d'un polymorphisme entre 2 lignées car, quel que soit l'oligonucléotide employé, l'électrophorèse est réalisée en gel d'agarose et la révélation consiste en une simple coloration au BrEt des fragments d'ADN amplifiés. Ceci permet d'analyser avec un même gel des amplifications obtenues avec des amorces différentes

La présence d'un appareil de PCR semble de plus en plus indispensable au sein d'un laboratoire de sélection. En effet, les applications de cette technique ne cessent d'augmenter dans le domaine de l'amélioration des plantes, la sélection assistée par marqueurs moléculaires devenant une pratique courante. De plus, des kits d'amorces pour la détection et la reconnaissance d'agents phytopathogènes apparaîtront prochainement sur le marché (Schesser et al, 1991).

\section{Quelques éléments de comparaison entre les techniques RAPD et RFLP}

Au niveau moléculaire, la méthode RAPD se distingue de la RFLP par une technologie plus 
simple et plus rapide, car elle ne fait pas intervenir de digestion enzymatique, de transfert sur membrane, d'hybridation avec une sonde marquée et de révélation. D'autre part, cette nouvelle technique nécessite très peu d'ADN matrice: $0,025 \mu \mathrm{g} /$ échantillon, contre $10 \mu \mathrm{g} /$ échantillon en RFLP. De plus, la très faible purification de I'ADN constitue un autre avantage de la RAPD sur la RFLP, pour laquelle la digestibilité de l'ADN par des enzymes de restriction est une condition indispensable. Il faut également souligner que les taux de réussite que nous avons obtenus pour distinguer les lignées parentales de nos différents croisements (voir plus haut) sont rarement atteints avec des sondes RFLP. En effet, afin de caractériser rapidement la lignée parentale mâle d'un hybride $F_{1}$, il est préférable d'obtenir des empreintes génétiques comportant plusieurs bandes au sein d'un même profil. C'est le cas général avec les amorces RAPD alors que seules quelques types de sondes RFLP génèrent de telles empreintes (sondes du phage M13, Rogstad et al, 1988, sondes minisatellites, Dallas, 1988, sondes synthétiques de répétitions en tandem, Mariat and Vergnaud, 1992, sondes d'ADN ribosomiques, etc).

Les objectifs de la technique RAPD sont les mêmes que ceux de la RFLP. II s'agit d'associer, chez les plantes cultivées, des marqueurs moléculaires à des caractères agronomiques favorables, ainsi facilement repérables dans la descendance des croisements intéressants. Cette démarche accélère considérablement le travail du sélectionneur. La généralisation de ces marqueurs chez une plante débouche sur la cartographie de son génome. Ces 2 techniques permettent alors l'obtention de véritables cartes d'identité génétique des plantes cultivées.

Les marqueurs RAPD présentent l'inconvénient majeur d'être dominants, contrairement à ceux issus de la RFLP. En effet, dans le second cas, une bande caractérisant une lignée sur l'autoradiogramme correspond à une autre bande spécifique d'une deuxième lignée révélant ainsi 2 allèles d'un même locus. Ceci résulte du fait qu'il est très peu probable, au sein d'une espèce, qu'une séquence complémentaire de la sonde ait totalement disparu d'un chromosome au cours de l'évolution. La dominance des marqueurs RAPD est liée à la technique elle-même. En effet, le polymorphisme est généralement dû à une recombinaison ayant affecté I'ADN d'une lignée, empêchant ainsi l'amplification d'un fragment d'ADN qui est réalisée avec l'ADN d'une autre lignée. C'est le cas si l'un des sites de fixa- tion de l'oligonucléotide disparaît par mutation (mutation ponctuelle, crossing-over, insertion, délétion, etc); si une insertion, n'affectant pas les 2 sites d'hybridation, éloigne ces derniers d'une distance ne permettant plus l'amplification d'un fragment; si une délétion rapproche les 2 sites de fixation de l'amorce, à l'origine d'un très petit fragment amplifié n'apparaissant pas sur le gel d'agarose. II est alors impossible de distinguer les individus hétérozygotes de ceux homozygotes pour le locus du marqueur RAPD. Toutefois, la codominance de marqueurs RAPD a été parfois observée (Williams et al, 1990). C'est le cas si un fragment amplifié chez une lignée correspond à un locus qui a subi, chez une lignée voisine, une courte insertion ou délétion (n'affectant pas les sites de l'amorce). Ceci est à l'origine d'un fragment amplifié de taille différente du premier et qui est visible sur le gel d'agarose.

Afin de contourner le problème de la dominance des marqueurs RAPD, il est préférable, pour les cartographier, d'utiliser les plantes issues du rétrocroisement de la $F_{1}$ avec l'une de ses lignées parentales ou issues de culture in vitro de cellules de la $F_{1}$ ayant subi la méiose. Toutefois, il est tout de même possible de travailler avec une $F_{2}$ mais cela requiert alors, pour une même précision, l'analyse d'un plus grand nombre d'individus.

\section{Problème de la dominance des marqueurs RAPD pour la vérification de la pureté hybride $F_{1}$ d'un lot de semences}

La dominance des marqueurs RAPD peut présenter un risque d'erreur lors du contrôle de la pureté d'un lot de semences supposées hybrides $F_{1}$. En effet, il reste généralement une petite proportion d'hétérozygotie au sein du génome des lignées parentales des hybrides. Ainsi, il est théoriquement possible de caractériser la lignée parentale mâle par un marqueur RAPD amplifié au niveau d'un locus hétérozygote. Dans un tel cas et en l'absence d'une quelconque autofécondation résiduelle de la lignée parentale femelle (taux d'inbreds nul), seulement la moitié des hybrides $F_{1}$ présenteront ce marqueur! Nous avons pu exclure cette interprétation pour notre hybride $F_{1 G M S}$, bien que le taux d'inbreds déterminé par RAPD fût proche de $50 \%$, car ce taux a été confirmé par un test phénotypique portant à la fois sur un autre lot de plantes de ce même hybride et sur les plantes 
soumises préalablement au test moléculaire. Afin d'éviter ce risque d'erreur, nous préconisons la pollinisation manuelle de quelques fleurs de la lignée femelle par du pollen de la lignée mâle pour une obtention non ambiguë de quelques semences hybrides $F_{1}$. II s'agit alors d'observer à l'aide de ces quelques plantes la ségrégation du marqueur RAPD de la lignée parentale mâle. Elle doit être de type 1:0 s'il y a homozygotie au niveau du locus d'amplification dans le génome de la lignée mâle ou de type 1:1 s'il y a hétérozygotie (dans ce dernier cas, 11 hybrides suffisent pour avoir $99 \%$ de chance d'observer au moins une plante homozygote ne présentant pas le marqueur).

Les résultats obtenus avec l'amorce D20 sur les hybrides $F_{1 O B S}$ (fig $5 b$ ) peuvent s'interpréter de 2 façons. Si la lignée $L_{5}$ est homozygote en ce qui concerne le locus de son marqueur RAPD, les 2 hybrides présentant ce marqueur sont les descendants de $L_{5}$ et les 3 autres sont ceux de $L_{6}$. Si $L_{5}$ est hétérozygote pour ce locus, les 2 hybrides marqués sont issus de $L_{5}$ et les autres peuvent aussi bien être les descendants de $L_{5}$ que de $L_{6}$. Nous n'avons pas pu trancher entre ces deux interprétations car nous n'avions pas à notre disposition de descendants fiables de $L_{5}$.

\section{CONCLUSION}

Les travaux présentés ici ont montré l'intérêt de la technique RAPD pour le contrôle de la production de variétés hybrides $F_{1}$ de chou-fleur. En effet, après avoir adapté le protocole de Williams et al (1990) à ce matériel végétal, nous avons pu utiliser cette technique pour analyser la pureté hybride de 2 lots de semences. Pour le sélectionneur, l'avantage de cette analyse moléculaire est non seulement un gain de temps par rapport au test phénotypique classique par préculture d'un échantillon, mais également l'obtention de résultats sans ambiguitté. II serait possible de réduire encore un peu la durée des analyses en extrayant I'ADN à partir de graines et non plus de jeunes plants (E Margalé, comm pers).

Au-delà de cette simple application, la RAPD semble pouvoir devenir un outil très efficace pour l'obtention d'empreintes génétiques des variétés de chou-fleur. En effet, un ensemble de profils RAPD très distincts entre 2 lignées laisse supposer un éloignement génétique important entre ces dernières. II est alors envisageable de croiser des lignées présentant des empreintes très différentes afin de vérifier si l'on peut bénéficier d'un fort effet d'hétérosis.

Cette nouvelle technique permet, comme la RFLP, de cartographier le génome de l'espèce étudiée et d'en étiqueter des gènes gouvernant des caractères agronomiques intéressants. $\mathrm{Ce}$ type de démarche devrait permettre une sélection assistée par des marqueurs moléculaires. Le travail du sélectionneur serait alors facilité et accéléré grâce à un diagnostic possible à un stade très précoce de développement de la plante, avant même l'expression du caractère étudié.

La méthode RAPD est donc destinée à devenir un outil de sélection végétale fiable et efficace. Mais elle peut également s'avérer très utile dans une démarche beaucoup plus fondamentale visant une approche phylogénique, grâce aux empreintes génétiques qu'elle fournit.

\section{REMERCIEMENTS}

Les auteurs tiennent à remercier Mlle L Boucault, M E Margalé et les Drs $R$ Dumas de Vaulx et $Y$ Hervé pour leur lecture critique de ce manuscrit. Ces travaux ont été financés avec l'aide du Conseil régional de Bretagne (programme Britta) et du Conseil général du Finistère. Ils ont bénéficié en outre d'un financement du ministère de la Recherche et de la Technologie dans le cadre du programme Eureka «RFLP Brassica EU324/F250".

\section{RÉFÉRENCES}

Arus P, Orton TJ (1983) Inheritance and linkage relationships of isozyme loci in Brassica oleracea. J Hered 74, 405-412

Arus P, Tanksley SD, Orton TJ, Jones RA (1982) Electrophoretic variation as tool for determining seed purity and breeding hybrid varieties of Brassica oleracea. Euphytica 31, 417-428

Berthomieu P, Meyer C (1991) Direct amplification of plant genomic DNA from leaf and root pieces using PCR. Plant Mol Biol 17, 555-557

Boucault L (1990) Étude des caractères de plantes issues d'androgenèse chez le chou-fleur d'automne (Brassica oleracea var botrytis). DEA de sci technol prod vég et agro-alim, Univ de Rennes I

Caetano-Anollés G, Bassam BJ, Gresshoff PM (1991) DNA amplification fingerprinting using very short arbitrary oligonucleotide primers. Biotechnology 9, 553-557

Carlson JE, Tulsieram LK, Glaubitz JC, Luk VWK, Kauffeldt C, Rutledge R (1991) Segregation of ran- 
dom amplified DNA markers in $F_{1}$ progeny of conifers. Theor App/ Genet 83, 194-200

Dallas JF (1988) Detection of DNA "fingerprints" of cultivated rice by hybridization with a human minisatellite DNA probe. Proc Natl Acad Sci USA 85, 68316835

Dellaporta SL, Woods J, Hicks JB (1983) A plant DNA minipreparation. Plant Mol Biol Rep 1, 19-21

Edwards K, Johnstone C, Thompson C (1991) A simple and rapid method for the preparation of plant genomic DNA for PCR analysis. Nucleic Acids Res 19,1349

Erlich HA, Saiki RK, Gelfand DH, Oste C, Higuchi R (1989) Part one : basic methodology. In: PCR technology, principle and applications for DNA amplification (Erlich HA, ed) M Stockton Press, 1-38

$\mathrm{Hu}$ J, Quiros CF (1991) Identification of brocoli and cauliflower cultivars with RAPD markers. Plant Cell Rep 10, 505-511

Klein-Lankhorst RM, Vermunt A, Weide R, Liharska T, Zabel P (1991) Isolation of molecular markers for tomato ( $L$ esculentum) using random amplified polymorphic DNA (RAPD). Theor Appl Genet 83, 108114

Mariat D, Vergnaud G (1992) Detection of polymorphic loci in complex genomes with synthetic tandem repeats. Genomics 12, 454-458

Nasrallah JB, Nishio T, Nasrallah ME (1991) The selfincompatibility genes of Brassica: expression and use in genetic ablation of floral tissues. Annu Rev Plant Physiol Plant Mol Biol 42, 393-422

Quiros CF, Hu J, This P, Chevre AM, Delseny M (1991) Development and chromosomal localization of genome-specific markers by polymerase chain reaction in Brassica. Theor Appl Genet 82, 627-632
Rogstad SH, Patton II JC, Schaal BA (1988) M13 repeat probe detects DNA minisatellite-like sequences in gymnosperms and angiosperms. Proc Natl Acad Sci USA 85, 9176-9178

Sambrook J, Fritsch EF, Maniatis T (1989) Molecular cloning, a laboratory manual. Cold Spring Harbor Laboratory Press, 2nd edn

Schesser K, Luder A, Henson JM (1991) Use of polymerase chain reation to detect the take-all fungus, Gaeumannomyces graminis, in infected wheat plants. Appl Environ Microbiol 57, 553-556

Tanksley SD, Orton TJ (1983) Isozymes in plant genetics and breeding. Elsevier, Amsterdam

Tanksley SD, Young ND, Paterson AH, Bonierbale MW (1989) RFLP mapping in plant breeding : new tools for an old science. Biotechnology 7, 257-264

Welsh J, McClelland M (1990) Fingerprinting genomes using PCR with arbitray primers. Nucleic Acids Res 18, 7213-7218

Welsh J, McClelland M (1991) Genomic fingerprinting using arbitrarily primed PCR and a matrix of pairwise combinations of primers. Nucleic Acids Res 19, 5275-5279

Welsh J, Petersen C, McClelland M (1991a) Polymorphisms generated by arbitrarily primed PCR in the mouse : application to strain identification and genetic mapping. Nucleic Acids Res 19, 303-306

Welsh J, Honeycutt RJ, McClelland M, Sobral BWS (1991b) Parentage determination in maize hybrids using the arbitrarily primed polymerase chain reaction (AP-PCR). Theor Appl Genet 82, 473-476

Williams JGK, Kubelik AR, Livak KJ, Rafalski JA, Tingey SV (1990) DNA polymorphisms amplified by arbitrary primers are useful as genetic markers. $\mathrm{Nu}$ cleic Acids Res 18, 6531-6535 